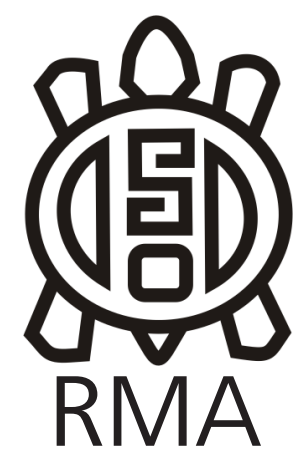

Arqueología

\title{
El uso de Rhea americana por los antiguos pobladores del sitio Beltrán Onofre Banegas-Lami Hernández del Chaco Seco (Santiago del Estero)
}

\author{
The use of Rhea americana by the ancient settlers of the Beltrán Onofre \\ Banegas-Lami Hernández archaeological site of the Dry Chaco (Santiago \\ Del Estero Province)
}

Luis M. del Papa* y Leda Moro**

*Cátedra de Anatomía Comparada, Facultad de Ciencias Naturales y Museo, UNLP, CONICET,

Argentina. E-mail: loesdelpapa@hotmail.com

** Facultad de Ciencias Naturales y Museo, UNLP, Argentina. E-mail:ledamoro@gmail.com

\begin{abstract}
Resumen
El rol de Rhea americana en la subsistencia de los antiguos pobladores ha sido analizado en diferentes regiones del país, principalmente en la Pampeana y Patagónica. En varios sitios de la provincia de Santiago del Estero se registra la presencia de R. americana con evidencias directas de consumo, y habría ocupado el segundo o tercer puesto de importancia en los recursos faunísticos de los grupos humanos luego de los camélidos para el período agroalfarero. En este trabajo se analizan los restos de R. americana del sitio Beltrán Onofre Banegas-Lami Hernández (contexto Sunchitúyoj, período agroalfarero tardío, ca. 800-300 AP) con el fin de evaluar los procesos que generaron su registro. Se realizó la cuantificación, la comparación con marcos de referencia y el análisis tafonómico de los materiales. En este sentido, se observa una mayor proporción de los elementos del miembro posterior, en menor medida del esqueleto axial y escaso del miembro anterior. Estos restos presentan evidencias de acumulación antrópica y una incidencia de la acción de agentes naturales que consideramos no fue significativa. De los resultados obtenidos, se infiere que la actividad antrópica fue el principal factor que contribuyó a una proporción diferencial de partes esqueletarias.
\end{abstract}

Palabras Clave: Rhea americana; Región Chaco-Santiagueña; Partes Esqueletarias; Tafonomía; Marcos de Referencia

\begin{abstract}
The role of Rhea americana in the subsistence of the ancient settlers has been analyzed in different regions of the country, mainly in Pampeana and Patagónica regions. The presence of $R$. americana remains with evidence of human consumption was registered in several archaeological sites of Santiago del Estero province. This species would have been second or third in order of importance in the diet of ancient human groups after camelids in the agro-pottery period. In this paper, the R. Americana remains of the Beltrán Onofre Banegas-Lami Hernández archaeological site (Sunchitúyoj context, late agro-pottery period, ca. 800-300 BP) are analyzed in order to evaluate the processes that generated its record. Quantification, comparison with frames of reference and taphonomic analysis of materials were performed. In this sense, a higher proportion of hindlimb elements, a lesser extent of the axial skeleton and a low proportion of forelimb are observed. These remains show evidence of anthropic accumulation and we considered that the incidence of the natural agents' action was not significant. From the results, it follows that human activities were the main factor contributing to a differential skeletal parts representation.
\end{abstract}

Keywords: Rhea americana; Chaco-Santiagueña Archaeological Region; Skeletal Parts; Taphonomy; Frames of Reference

En tiempos prehispánicos los reidos (Rhea americanañandú-, Pterocnemia pennata-ñandú petiso, suri o choique-) han sido utilizados como recurso (alimentos, plumas, cueros, materia prima en la confección de artefactos, etc.) dentro de las prácticas de los antiguos pobladores de diferentes regiones de la Argentina, principalmente de la región Pampeana y la Patagónica (e.g.Acosta 2005; Álvarez 2015; Cornaglia Fernández 2013; del Papa 2012; Fernández 2000, 2010; Frontini y Picasso 2010; Giardina 2010; Miotti 1998; Quintana y Mazzanti 2001; Santini 2009), llegando incluso a mantenerse durante gran parte del período postcontacto (e.g. Cieza de León 1548-1551 [1877]; Fernández 1571 [1914]; Lizarraga ca. 1604-1607 [1999]; Sánchez Labrador 1910; Sotelo de Narváez 1583 [1885]; véase Salemme y Frontini 2011 para Pampa y Patagonia entre otros). De los distintos análisis se ha interpretado el uso de Rheidae tanto por la obtención de nutrientes (carne, médula, grasa, huevos), de tendones, cueros (e.g. Bonomo et al. 2008; del Papa 2012; Fernández 2010; Frontini y 
Picasso 2010; Giardina 2010; González de Prado 15481556 [1919]; Medina et al. 2011; Miotti 1998, Miotti y Salemme 1999; Prates y Acosta Hospitaleche 2010; Quintana y Mazzanti 2001; Salemme y Frontini 2011; entre otros), los huevos como contenedores y un rol decorativo y simbólico (e.g. Carden y Martínez 2014; Fiore y Borella 2010), las plumas para vestimenta u ornamento (e.g. Cieza de León 1548-1551 [1877]; Fernández 1571 [1914]; Miotti y Salemme 1999; Sotelo de Narváez 1583 [1885]) y el uso de sus huesos como materia prima en la confección de artefactos (e.g. Pérez Jimeno y del Papa 2016; Rusconi 1933; Sánchez Labrador 1910). Por su parte, Sotelo de Narváez (1583 [1885]) menciona la cría de ñandú por parte de los indígenas en la gobernación de Tucumán, cuya ciudad cabecera para ese momento (1583) era Santiago del Estero: "Criaban avestruces mansos en sus casas, gallinas y patos, y asi lo hacen ahora, aunque después que los xpianos entraron en aquella tierra, se visten todos en general, a fuer de los del Pirú, de lana y algodón." (Pedro Sotelo de Narváez 1583[1885]: 143-153, resaltado nuestro). Sin embargo, tanto en las fuentes etnohistóricas de la primera entrada de los españoles en territorio santiagueño, hasta posteriores a Sotelo de Narváez, ésta es la única referencia a la cría de ñandú, por lo que se infiere que la misma podría haberse dado como una consecuencia de la intervención de los españoles generando así la modificación del manejo de los animales por parte de los nativos.

En los sitios arqueológicos de la región Pampeana y Patagónica se observó un patrón diferencial de partes esqueletarias de Rheidae, donde predominan los elementos del miembro posterior, mientras que los elementos axiales y del miembro anterior son escasos o nulos (e.g. Belardi 1999; Belardi y Carballo Marina 2003; Cruz 2011; Cruz y Elkin 2003; Fernández 2010; Fernández et al. 2001; Frontini y Picasso 2010). Este patrón se ha analizado desde distintos enfoques, como los estudios actualísticos sobre la depositación y la preservación de carcasas, y estudios tafonómicos de sitios arqueológicos atribuyéndolo a distintos factores. Entre éstos se pueden mencionar la destrucción diferencial (mediada por la densidad mineral ósea) por procesos naturales (e.g. acción de carnívoros, meteorización; Belardi 1999; Belardi y Carballo Marina 2003; Cruz 2011; Cruz y Elkin 2003; Fernández 2010; Fernández et al. 2001), o a decisiones humanas en el transporte de las partes a ser consumidas (inferido a partir de índices de utilidad económica, Giardina 2006). Sin embargo, en los resultados obtenidos por Giardina (2006: 271) en la correlación de los valores de densidad mineral ósea y los índices de utilidad, se observa un valor que no es despreciable, por lo que señala un cierto grado de equifinalidad entre las partes de mayor rendimiento y las de mayor densidad mineral.

Para la provincia de Santiago del Estero (región ChacoSantiagueña), en varios sitios arqueológicos se ha registrado la presencia de $R$. americana, en la mayoría de los casos con evidencias directas de consumo (del Papa 2012). Cabe mencionar que según el estimador de biomasa (MNI multiplicado el peso promedio del taxón en vida) calculado en los sitios, se infiere que este recurso habría estado en el segundo o tercer puesto de importancia en la dieta de los grupos humanos luego de los camélidos (del Papa 2012; del Papa y Togo 2015; del Papa et al. 2012). En este sentido, y como objetivo general de este trabajo, se considera importante analizar el rol de $R$. americana en la subsistencia de los antiguos pobladores del sitio Beltrán Onofre Banegas-Lami Hernández (de ahora en adelante se nombrará como BOL), y como objetivos particulares, evaluar los procesos que intervinieron en la conformación de la muestra y las prácticas sociales asociadas a la utilización de esta especie (obtención, procesamiento, consumo, etc.).

\section{Descripción del taxón}

La familia Rheidae está conformada por aves corredoras que incluyen a las especies Rhea americana y Pterocnemia pennata. $R$. americana es el ave más grande del continente (15-40 kg) y se distribuye desde el norte de Brasil hasta el norte de la Patagonia argentina (Martella y Navarro 2006), incluyendo la zona de estudio de este trabajo. A su vez, P. pennata (15-27 kg) habita el sur de Perú, sudoeste de Bolivia, Chile y la región oeste y la patagónica de Argentina (Martella y Navarro 2006). $R$. americana es una especie neotropical que habita exclusivamente en las planicies abiertas poco arboladas y generalmente busca lugares cercanos a ríos, lagos o estanques, donde la comida es abundante. Se alimenta principalmente de semillas, raíces, hojas, insectos y vertebrados pequeños (Gómez de Silva et al. 2005). Son aves de hábitos diurnos y comportamiento gregario en la época no reproductiva, formando grupos desde 2-3 individuos hasta bandas de más de 50. La composición de estos grupos puede ser variable, esto es, solamente machos, machos y hembras o bien, machos, hembras y juveniles del año anterior (Reboreda y Fernández 2005). El período de reproducción es entre agosto y enero y hacia fines de agosto se produce la fragmentación de los grupos invernales cuando un macho dominante monopoliza y defiende, mediante la exclusión de otros machos, un grupo de hembras (Martella y Navarro 2006; Gómez de Silva et al. 2005). El macho dominante construye un nido (depresión en el suelo de $1 \mathrm{~m}$ de diámetro, forrada de pasto seco y ramas) y copula con las hembras de su harén, las que ponen sus huevos en forma comunal a intervalos de dos a tres días. El número de huevos puede variar entre 20 y 50 por nidada (Reboreda y Fernández 2005). La incubación se extiende por 36-37 días, y a pesar de las diferencias temporales que existen en la puesta de los huevos, la eclosión de estos es sincrónica. El macho no sólo es el encargado de la incubación sino también del cuidado de los pichones (Reboreda y Fernández 2005). Tan pronto como el período de reproducción finaliza, se vuelven a formar grupos integrados por jóvenes y 
adultos (Folch 1992). El comportamiento diurno, gregario y frecuentar zonas cercanas a fuentes de agua convierte al ñandú en un recurso predecible para la subsistencia humana (Giardina 2006).

Debido al hábito corredor, los Rheidae presentan un gran desarrollo del miembro posterior, esto es, los huesos de las extremidades posteriores (fémur, tibio-tarso, tarsometatarso y falanges) poseen paredes gruesas y valores más altos de densidad mineral ósea (Reboreda y Fernández 2005; Cruz y Elkin 2003). Por su parte, las extremidades anteriores, el cráneo y el esternón presentan una baja densidad mineral ósea por lo que podrían destruirse más rápidamente que las extremidades posteriores ante diversos procesos destructivos (Cruz 2011).

\section{Sitio Beltrán Onofre Banegas-Lami Hernández (BOL)}

El sitio arqueológico BOL (Dpto. Robles, Santiago del Estero), está ubicado a $27^{\circ} 49^{\prime} 08^{\prime \prime}$ de latitud sur y $64^{\circ} 02^{\prime} 43^{\prime \prime}$ de longitud oeste, aproximadamente a $1 \mathrm{~km}$ del sitio Beltrán Cementerio de igual contexto cultural (Figura 1). BOL se integra por su localización al espacio subtropical semiárido y continental del país. Las temperaturas promedio para la zona de estudio tienen valores de $20^{\circ} \mathrm{C}$, con precipitaciones que se concentran en el período estival de $550 \mathrm{~mm}$ anuales y capacidad de evapotranspiración potencial mayor, lo que determina una gran deficiencia hídrica (Ledesma 1979). Dicho sitio se integra en la Eco-región del Chaco Seco, subregión Chaco semiárido (Burkart et al. 1999), que se caracteriza por la presencia de un bosque xerófilo y semi caducifolio y fauna de origen brasílico o tropical, con elementos adaptables a condiciones rigurosas de sequedad (Ringuelet 1961).

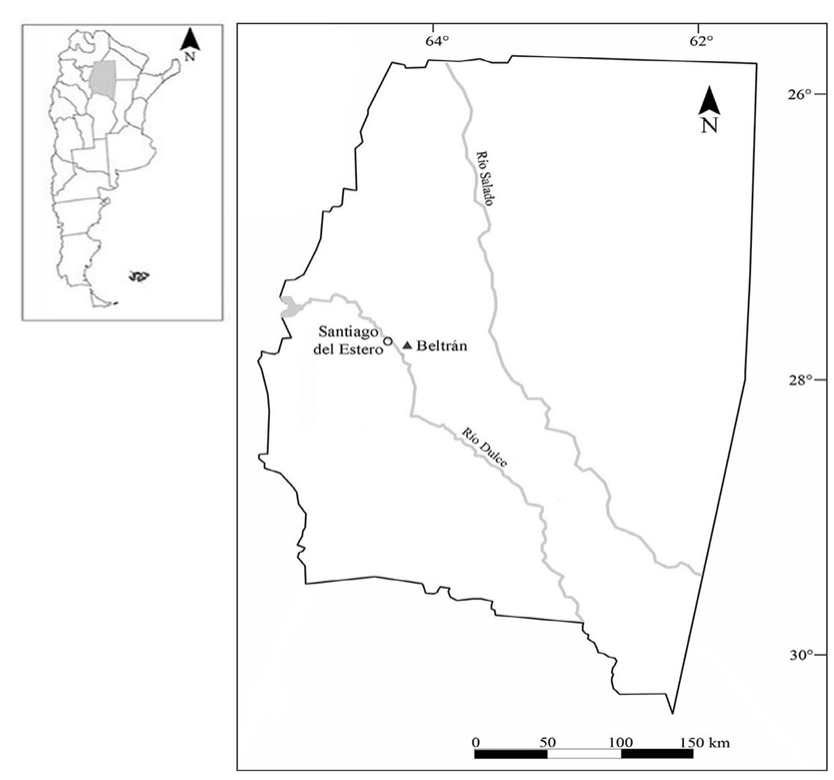

Figura 1. Ubicación del sitio Beltrán Onofre Banegas-Lami Hernández.

Figure 1. Beltrán Onofre Banegas-Lami Hernández site location.
El sitio BOL se ubica en la proximidad de un paleocauce del río Dulce y se distingue por la presencia de montículos de origen mixto (naturales y antrópicos). En este trabajo se tienen en cuenta los materiales procedentes de ocho cuadrículas y la extensión de dos de ellas (dispersas en dos montículos), con una superficie de 28,75 $\mathrm{m}^{2}$ (ver más detalles en del Papa y De Santis 2015: Figura 2). En la mayoría de las cuadrículas los restos arqueológicos se concentran entre los 10 y $50 \mathrm{~cm}$ (desde la superficie del terreno), reduciéndose drásticamente a partir de esta última profundidad. En la excavación no se distinguieron diferencias significativas en los sedimentos que contienen los restos arqueológicos, de composición limo-arenosa (del Papa y De Santis 2015).Como resultado de los trabajos de campo llevados a cabo en el sitio, se recuperó gran diversidad de material lítico, óseo y cerámico. Con respecto a éste último, luego de una observación preliminar, fue asignado al contexto agroalfarero tardío con presencia principalmente de fragmentos Sunchitúyoj y escasos restos Averías, aunque todavía se encuentran bajo estudio. Se obtuvo un fechado radiocarbónico a partir de muestras de carbón vegetal de $420 \pm 60$ años ${ }^{14}$ C AP (LP-2054) (del Papa y De Santis 2015).

\section{Contexto cultural}

El período agroalfarero tardío en la región ChacoSantiagueña (ca. 800-300 AP), está caracterizado por la presencia de grupos sedentarios portadores de la cerámica Sunchitúyoj y Averías (Togo 2004). La agrupación de Sunchitúyoj y Averías en la Tradición Cultural Chaco-Santiagueña por Lorandi (1978) se basa en que los sitios analizados presentan un patrón de asentamiento y un sistema económico con similares características, siendo que sus diferencias giran en torno al tipo decorativo y estilístico presente en la cerámica y a la intensidad de algunos procesos (e.g. mayor énfasis de las prácticas textiles y un aumento poblacional en Averías). Sin embargo, Togo (2004) considera útil mantener los nombres Sunchitúyoj y Averías en sentido amplio, ya que contemporáneamente se encuentran sitios puros sin asociación entre sí, lo que denotaría la presencia de grupos humanos independientes dentro del territorio provincial que elaboraron materiales con tecnología y simbolismos diferentes, a pesar de la semejanza en cuanto al patrón de asentamiento (construcciones habitacionales sobre montículos naturales, artificiales o mixtos), economía (mixta, agrícola-ganadera y cazadora-recolectora), funebria y cierta tecnología cerámica (compartirían los mismos tipos "ordinarios" de la cerámica y algunos elementos decorativos) (Lorandi 1974; Togo 2004). Según nuevos fechados disponibles, los grupos Sunchitúyoj se desarrollaron entre el 1200 y 1500 dC (Togo 2007) y podrían haber perdurado hasta la conquista española, por lo menos en algunas zonas de la provincia (Gramajo de Martínez Moreno 1978; Togo 2004). Por su parte, Averías se habría desarrollado muy cercano a la llegada de los conquistadores europeos, esto se desprende de las asociaciones directas con elementos hispánicos. 


\section{Materiales y Métodos}

El sitio BOL fue relevado y excavado en sucesivas campañas desde 2008 a 2010, recuperándose una gran cantidad de restos arqueofaunísticos (15947 especímenes). Del total de restos recuperados, 557 fueron asignados a $R$. americana (74 restos óseos y 483 fragmentos de cáscaras de huevo). Se analizaron los restos óseos de ñandú teniendo en cuenta los niveles de análisis tanto cuantitativo como cualitativo. En lo referente al primero, se incluyeron el NISP (number of identified specimens per taxon; Payne 1975) y MNI (minimum number of individuals) siguiendo criterios de lateralidad y edad (Bökönyi 1970). En el caso en que se producía el remontaje mecánico de los restos óseos (aquellos con fracturas secas y actuales), se consideró a los fragmentos que remontaban como un solo espécimen (Clason 1972; Reitz y Wing 1999), y de esta manera reducir el error en la cuantificación (del Papa 2010).

También se multiplicó el MNI por el peso promedio del taxón en vida a modo de estimar la biomasa obtenida de los diferentes recursos (véase del Papa 2012; del Papa y Togo 2015; del Papa et al. 2012). Por otra parte, se consideraron los cálculos de representación de partes esqueletarias, mediante el MNE (minimum number of elements) y el MAU\% (standardized minimum number of animal units) (Binford 1984; Mengoni Goñalons 1999). En el caso de las cáscaras de huevo, se siguió a Quintana (2007) a través del método comparativo para calcular el MNI partiendo del peso promedio de dichas cáscaras.

Con el fin de poder interpretar el registro particular de partes esqueletarias de $R$. americana, se realizaron correlaciones (a través del coeficiente de Spearman) entre la representación de partes esqueletarias (MAU\%) y diversos marcos de referencia de $P$. pennata (ñandú petiso), ya que, no hay índices disponibles para $R$. americana. En primer lugar, se correlacionó con los valores de densidad mineral ósea (DMO) calculados por Cruz y Elkin (2003) con el propósito de estimar la preservación diferencial mediada por esta variable. Luego se correlacionó con los índices de utilidad económica para inferir patrones de transporte, calculados por Giardina (2006): utilidad de carne (MUI), índice de grasa (WGUI), índice de utilidad general modificado (MGUI) e índice de médula (MI). Debido a que el MI sólo tiene en cuenta a los huesos largos, no sólo se correlacionó la DMO con la totalidad del perfil anatómico, sino también con los huesos largos en particular para comparar los resultados obtenidos de las diferentes variables. Por otra parte, según los resultados obtenidos en las correlaciones, se tuvieron en cuenta las clases propuestas por Lyman (1994: Figura 7.13) las cuales nos permiten explorar si el patrón anatómico es resultado de una selección humana para su transporte, si se encuentra sesgado por procesos naturales o si se está ante un caso de equifinalidad. Los resultados fueron comparados con casos de estudio de otras regiones.
Cabe destacar, que en los casos de equifinalidad, el análisis de las modificaciones óseas producidas por distintos procesos tafonómicos nos puede ayudar a interpretar el registro de partes esqueletarias (Fernández 2010). Para esto, se tuvieron en cuenta aquellos procesos naturales que pudieron haber generado la depositación de restos y la alteración del registro arqueológico, como por ejemplo los depredadores naturales, roedores, raíces, depositaciones químicas y meteorización (Binford 1981; Blumenschine et al. 1996; Lyman 1994; Muñoz y Savanti 1998). Para el análisis de la meteorización, se tuvieron en cuenta los estadios nominales propuestos por Muñoz y Savanti (1998) para aves voladoras y nadadoras, que van de 1 a 4 (E1 a E4), y se incorporó además el estadio 0 (E0= ausencia de atributos asociados a la meteorización).

Del mismo modo, con el fin de obtener información sobre los posibles patrones de procesamiento, cocción, consumo y descarte de los alimentos, se analizaron las modificaciones como las marcas de corte, machacado, fractura intencional, marcas de percusión, producción de artefactos y termoalteración (Binford 1981; Blumenschine et al. 1996; Mengoni Goñalons 1999; Stiner et al. 1995). Con respecto a las fracturas, se evaluó el estado del material óseo al momento de fracturación (estadios fresco, seco e intermedio) a través del índice de fractura fresca (Fracture Freshness Index, FFI) de Outram (2002). El FFI se basa en tres criterios: a- ángulo de la superficie de fractura, si son agudos u obtusos van a tener un valor de 0 y si son rectos un valor de 2 , siendo los ángulos intermedios, de valor 1 ; b- forma de la fractura, si son helicoidales corresponde un valor de 0 , si son oblicuas o una combinación de helicoidales/ transversales o helicoidales/longitudinales, un valor de 1 y si son transversales o longitudinales un valor de 2; y c- textura de la superficie de fractura, si es suave le corresponde un valor de 0 , si es rugosa valor de 2 y de rugosidad intermedia valor de 1 . Entonces, los valores para cada criterio son 0,1 , 2; donde el valor 0 es el que concuerda con la fractura en estado fresco; 1 cuando el espécimen tiene algún atributo del estado seco; y 2 cuando predomina el estado seco en la producción de la fractura. El índice, entonces va a variar de 0 a 6 . En este sentido, se identificaron fracturas en estado fresco (valores 0,1 y 2 del FFI), fracturas en estado intermedio, donde se produjo una cierta deshidratación del hueso o perdida de elasticidad previa a la fractura (valores 3 y 4) y fracturas en estado seco, donde el hueso perdió la mayoría o toda su elasticidad debido principalmente a factores diagenéticos o por procesos extremos (valores 5 y 6). Por último, se analizaron los artefactos óseos presentes en la muestra siguiendo los criterios utilizados por Scheinsohn (1997) y Pérez Jimeno (2004), quienes proponen la clasificación de las piezas en diferentes grupos de acuerdo con la estructura física (teniendo en cuenta la categoría taxonómica y el soporte), estructura morfológica y el tratamiento de la superficie. 


\section{Resultados}

En la Tabla 1 se puede observar la predominancia de restos de mamíferos NISP\% $=53,45$ (destacándose Chaetophractus vellerosus con el 7,21\% y Lama sp. con el 4,89\%), seguido por peces con 29,43 , luego aves con 9,33 , y en cuarto lugar reptiles con 5,87 . En el caso de R. americana, se destacan las cáscaras de huevo con un $\mathrm{NISP} \%=4,63$ y un peso de $131,8 \mathrm{gr}(\mathrm{MNI}=2)$, mientras que los restos óseos presentan un porcentaje menor (NISP\%= $0,68)$. Sin embargo, teniendo en cuenta el estimador MNI por peso promedio del taxón en vida, se puede observar la preponderancia de aquellos recursos que brindan una mayor cantidad de nutrientes, como es el caso de Lama sp. $(67,67 \%)$ y $R$. americana $(10,58 \%)$, quedando relegados a un segundo plano aquellos recursos de menor aporte nutricional.

\section{Representación de partes esqueletarias de Rhea americana}

En las Tablas 2 y 3 y en la Figura 2 se puede observar que hay una mayor proporción de los elementos del esqueleto apendicular posterior (principalmente tibio-tarso, seguido de peroné, tarso-metatarso, fémur y pelvis), y en un menor número los elementos del miembro anterior y esqueleto axial (húmero, vértebras y costillas). Este patrón de representación de partes no se correlaciona significativamente con la DMO (DMO vs MAU\%, rs=
0,$326 ; \mathrm{P}>0,05, \mathrm{~N}=30$ y $\mathrm{DMO}$ vs MAU\% miembros, $r s=0,159 ; \mathrm{P}>0,05, \mathrm{~N}=20)$ ni con el $\mathrm{MUI}(r s=0,137 ; \mathrm{P}>$ $0,05, \mathrm{~N}=23)$ ni el WGI $(r s=0,01 ; \mathrm{P}>0,05, \mathrm{~N}=23)$, pero si se correlaciona significativamente con el $\mathrm{MI}$ y el MGUI (MAU\% vs $\mathrm{MI}, r s=0,695 ; \mathrm{P}<0,05, \mathrm{~N}=15$ y $\mathrm{MAU} \%$ vs MGUI, $r s=0,64 ; \mathrm{P}<0,05, \mathrm{~N}=23$ ).

Siguiendo las clases de Lyman (1994) (Tabla 4) se observa que considerando la muestra general como los huesos largos en particular caen en la Clase 8, interpretándose como una estrategia Bulk o Gourmet en la selección de partes esqueletarias. Para otros casos de distintas regiones del país (Tabla 4), no se pudo comprobar el transporte selectivo a través de las correlaciones, ya que presentan muestras que se incluyen en la Clase 4 (muestras sesgadas por problemas de preservación), otras en la Clase 1, 4 o 7 (muestras que representan equifinalidad), y otras que representan la Clase 5 donde no se puede explicar el registro de partes esqueletarias por las variables analizadas (correlaciones no significativas tanto para DMO como para los índices de utilidad) (Álvarez 2015; Cruz y Elkin 2003; Frontini y Picasso 2010; Giardina 2010).

\section{Procesos naturales}

El estado de la superficie cortical de la muestra en general es bueno. La mayoría de los materiales presentan estadios bajos o nulos de meteorización (E0=59\% y E1= $33 \%)$, seguido por una baja presencia de estadios más avanzados (E2=8\%). Los escasos restos que presentan E2 corresponden a una porción proximal de diáfisis de tibio-tarso y los restantes a tarso-metatarso (dos mitades proximales y una porción distal). Para el caso de las aves, varios autores plantean que el predominio de estos estadios más bajos de meteorización, muchas veces se encuentra asociado a las propiedades mecánicas de los huesos de las aves que facilitan su destrucción antes de alcanzar estadios avanzados, sobre todo de aquellos de baja DMO (e.g. Behrensmeyer et al. 2003;

Tabla 1. Abundancia taxonómica (NISP, NISP\%, MNI y MNI multiplicado el peso promedio del taxón en vida). *incluye Tinamiformes (Eudromia elegans, Nothura sp.), Anatidae, Falco sparveirus, Geranoaetus melanoleucus, Tyto alba, Psittacidae, Columbiformes y Passeriformes; ${ }^{\circ}$ no se tienen en cuenta para el cálculo aquellos microvertebrados que por el análisis preliminar de sus restos podrían haber ingresado por muerte natural y/o depredadores naturales.

Table 1. Taxonomic abundance (NISP, NISP\%, MNI and MNI multiplied the average weight of the taxon in life).

*It includes: Tinamiformes (Eudromia elegans, Nothura sp.), Anatidae, Falco sparveirus, Geranoaetus melanoleucus, Tyto alba, Psittacidae, Columbiformes and Passeriformes; ' ${ }^{\circ}$ those microvertebrates that by $a$ preliminary analysis of their remains might have died a natural death and/or have been hunted by natural predators are not taken into account. 


\begin{tabular}{lcccc}
\hline Unidad anatómica & NISP & MNE & Enteros & $\%$ enteros \\
\hline Cráneo & 0 & - & - & - \\
Mandíbula & 0 & - & - & - \\
Vértebra cervical & 12 & 6 & 4 & 33,33 \\
Costilla, porción esternal & 1 & 1 & - & - \\
Costilla, porción vertebral & 3 & 3 & - & - \\
Escápula & 0 & - & - & - \\
Coracoides & 0 & - & - & - \\
Esternón & 0 & - & - & - \\
Húmero & 1 & 1 & - & - \\
Radio & 0 & - & - & - \\
Ulna & 0 & - & - & - \\
Carpo-metacarpo & 0 & - & - & - \\
Pelvis & 4 & 2 & - & - \\
Fémur & 4 & 3 & 1 & 25 \\
Tibio-tarso & 21 & 8 & - & - \\
Peroné & 9 & 4 & - & - \\
Tarso-metatarso & 11 & 4 & - & - \\
Falange proximal (miembro posterior) & 2 & 2 & 2 & 100 \\
Falange medial(miembro posterior) & 3 & 3 & 3 & 100 \\
Diáfisis indet., fragmento & 3 & - & - & - \\
\hline Total & 74 & 37 & 10 & 13,51 \\
\hline
\end{tabular}

Figura 2. Representación de partes esqueletarias de Rhea americana.

Figure 2. Skeletal parts representation of Rhea americana.
Tabla 2. Abundancia de partes esqueletarias (NISP, MNE) y porcentaje de especímenes enteros.

Table 2. Skeletal parts representation (NISP, MNE) and percentage of whole specimens.

\begin{tabular}{|c|c|c|c|}
\hline Elemento & MNE & MAU & MAU\% \\
\hline Cráneo & 0 & 0 & 0 \\
\hline Mandíbula & 0 & 0 & 0 \\
\hline Vértebra cervical & 6 & 0,5 & 12,5 \\
\hline Vértebra torácica & 0 & 0 & 0 \\
\hline Costilla, porción esternal & 1 & 0,125 & 3,125 \\
\hline Costilla, porción vertebral & 3 & 0,083 & 2,075 \\
\hline Escápula & 0 & 0 & 0 \\
\hline Coracoides & 0 & 0 & 0 \\
\hline Esternón & 0 & 0 & 0 \\
\hline Húmero, porción proximal & 0 & 0 & 0 \\
\hline Húmero, diáfisis & 1 & 0,5 & 12,5 \\
\hline Húmero, porción distal & 0 & 0 & 0 \\
\hline Radio & 0 & 0 & 0 \\
\hline Ulna & 0 & 0 & 0 \\
\hline Carpo-metacarpo & 0 & 0 & 0 \\
\hline Pelvis & 2 & 1 & 25 \\
\hline Fémur, porción proximal & 2 & 1 & 25 \\
\hline Fémur, diáfisis & 2 & 1 & 25 \\
\hline Fémur, porción distal & 2 & 1 & 25 \\
\hline Tibio-tarso, porción proximal & 2 & 1 & 25 \\
\hline Tibio-tarso, diáfisis & 8 & 4 & 100 \\
\hline Tibio-tarso, porción distal & 2 & 1 & 25 \\
\hline Peroné, porción proximal & 4 & 2 & 50 \\
\hline Peroné, cuerpo & 3 & 1,5 & 37,5 \\
\hline Tarso-metatarso, porción proximal & 2 & 1 & 25 \\
\hline Tarso-metatarso, diáfisis & 3 & 1,5 & 37,5 \\
\hline Tarso-metatarso, porción distal & 4 & 2 & 50 \\
\hline Falange proximal (miembro posterior) & 2 & 0,33 & 8,25 \\
\hline Falange medial (miembro posterior) & 3 & 0,25 & 6,25 \\
\hline Falange distal (miembro posterior) & 0 & 0 & 0 \\
\hline
\end{tabular}

Table 3. Representación de partes esqueletarias (MNE, MAU y MAU\%).

Table 3. Skeletal parts representation (MNE, MAU and MAU\%).
Belardi 1999; Cruz 2011, 2015; Muñoz y Savanti 1998). Cabe mencionar que los elementos de menor DMO representados en la muestra (e.g. costillas, vértebras y porción proximal de fémur, véase Cruz y Elkin 2003) presentan en su mayoría estadios nulos (E0) y escasos estadios bajos (E1) de meteorización, siguiendo la tendencia de los restantes elementos. En este sentido, el perfil de meteorización nos indica que la mayoría de los restos se enterraron relativamente rápido. En cuanto a la alteración de la superficie ósea producida por otros procesos naturales, se puede observar que la mayor parte de las modificaciones fueron producidas por precipitaciones químicas (50\%) a modo de pequeñas manchas de óxido de manganeso, seguidos por raíces $(35,13 \%)$, y en última instancia, roedores (14,86\%). Estas modificaciones podrían estar enmascarando otras marcas preexistentes (e.g. marcas de corte). La incidencia de roedores y raíces nos sugiere el desplazamiento espacial de los materiales dentro del depósito sedimentario, también evidenciada por la actividad de animales fosoriales registrada en el sitio (del Papa et al. 2017). Es importante destacar que en la muestra no se observaron evidencias de alteraciones producidas por depredadores naturales.

Con respecto a la fragmentación, se realizó el remontaje mecánico asociado a fracturas postdepositacionales, con superficies ásperas de color diferente al del resto del espécimen en el 79,72\% de los casos (59 fragmentos). A esto se asocia un bajo porcentaje de elementos enteros (Tabla 2), y sólo las falanges (huesos cortos) están completas. Del total de los huesos largos determinados en la muestra, el $62,50 \%$ se encuentra fracturado. De este porcentaje, la mayor proporción se produjo con el hueso en estado seco (60\%), el $20 \%$ en estado fresco y el restante $20 \%$ en estado intermedio (cuando el hueso perdió algo de elasticidad). Como se mencionó anteriormente, los factores que ocasionaron la mayoría de las fracturas producidas en estado seco, 


\begin{tabular}{|c|c|c|c|c|c|c|c|c|}
\hline Sitio & Región & Muestra & NISP & DMO & $\begin{array}{c}\text { Índices de } \\
\text { utilidad. }\end{array}$ & $\begin{array}{c}\text { Clases } \\
\text { de } \\
\text { Lyman } \\
(1994)\end{array}$ & Interpretación & Referencia \\
\hline $\mathrm{BOL}$ & $\begin{array}{c}\text { Chaco- } \\
\text { Satiagueña }\end{array}$ & $\begin{array}{c}\text { Muestra } \\
\text { general }\end{array}$ & 74 & $\begin{array}{c}+, \text { no } \\
\text { significativo }\end{array}$ & $\begin{array}{c}\text { MGUI (+, } \\
\text { significativo) }\end{array}$ & 8 & $\begin{array}{l}\text { Bulk o } \\
\text { Gourmet }\end{array}$ & $\begin{array}{c}\text { Este } \\
\text { trabajo }\end{array}$ \\
\hline $\mathrm{BOL}$ & & $\begin{array}{l}\text { Elementos } \\
\text { de los } \\
\text { miembros }\end{array}$ & 48 & $\begin{array}{c}+, \text { no } \\
\text { significativo }\end{array}$ & $\begin{array}{c}\text { MI (+, } \\
\text { significativo) }\end{array}$ & 8 & $\begin{array}{l}\text { Bulk o } \\
\text { Gourmet }\end{array}$ & $\begin{array}{l}\text { Este } \\
\text { trabajo }\end{array}$ \\
\hline $\mathrm{PO} 4$ & Pampeana & $\begin{array}{l}\text { Muestra } \\
\text { general }\end{array}$ & 27 & $\begin{array}{c}+, \text { no } \\
\text { significativo }\end{array}$ & - & - & - & $\begin{array}{l}\text { Álvarez } \\
\text { (2015) }\end{array}$ \\
\hline $\begin{array}{l}\text { El } \\
\text { Guanaco }\end{array}$ & Pampeana & $\begin{array}{l}\text { Muestra } \\
\text { general }\end{array}$ & 17 & $\begin{array}{c}-, \text { no } \\
\text { significativo }\end{array}$ & $\begin{array}{c}+, \text { no } \\
\text { significativo }\end{array}$ & 5 & - & $\begin{array}{l}\text { Frontini y } \\
\text { Picasso } \\
(2010)\end{array}$ \\
\hline $\begin{array}{l}\text { Parque } \\
\text { Nacional } \\
\text { perito } \\
\text { Moreno }\end{array}$ & Patagonia & $\begin{array}{c}\text { Muestra } \\
\text { general } \\
\text { actualistica }\end{array}$ & - & $\begin{array}{c}+, \\
\text { significativo }\end{array}$ & - & $1,4 \circ 7$ & $\begin{array}{l}\text { Sesgada, o } \\
\text { equifinalidad }\end{array}$ & $\begin{array}{l}\text { Cruz y } \\
\text { Elkin } \\
(2003)\end{array}$ \\
\hline CCe1 & Patagonia & $\begin{array}{l}\text { Muestra } \\
\text { general }\end{array}$ & 43 & $\begin{array}{c}+, \\
\text { significativo }\end{array}$ & - & $1,4 \circ 7$ & $\begin{array}{l}\text { Sesgada, o } \\
\text { equifinalidad }\end{array}$ & $\begin{array}{l}\text { Cruz y } \\
\text { Elkin } \\
(2003)\end{array}$ \\
\hline $\mathrm{PC} 1$ & Patagonia & $\begin{array}{l}\text { Muestra } \\
\text { general }\end{array}$ & 27 & $\begin{array}{c}+, \text { no } \\
\text { significativo }\end{array}$ & $\begin{array}{c}+, \text { no } \\
\text { significativo }\end{array}$ & 5 & - & $\begin{array}{l}\text { Giardina } \\
(2010)\end{array}$ \\
\hline PC2 & Patagonia & $\begin{array}{l}\text { Muestra } \\
\text { general }\end{array}$ & 25 & $\begin{array}{c}+, \\
\text { significativo }\end{array}$ & $\begin{array}{c}+, \text { no } \\
\text { significativo }\end{array}$ & 4 & Sesgada & $\begin{array}{l}\text { Giardina } \\
(2010)\end{array}$ \\
\hline
\end{tabular}

Tabla 4:.Comparación entre el caso de estudio y otras regiones. Correlaciones con marcos de referencia y clases de Lyman asociados (Lyman 1994). BOL: Beltrán Onofre Banegas-Lami Hernández; PO4: Paso Otero 4; CCe 1: Campo Cerda 1; PC1: Alero Puesto Carrasco, unidad litoestratigráfica 1; PC2: Alero Puesto Carrasco, unidad litoestratigráfica 2.

Table 4. Comparison between the study case and other regions. Correlations with frameworks of reference and Lyman's classes (Lyman 1994). BOL: Beltrán Onofre Banegas-Lami Hernández; PO4: Paso Otero 4; CCe 1: Campo Cerda 1; PC1: Alero Puesto Carrasco, lito-stratigraphic unit 1; PC2: Alero Puesto Carrasco, lito-stratigraphic unit 2.

probablemente fueron los procesos de deshidratación postdepositacional por diagénesis. Uno de los indicadores de la fragmentación por diagénesis es la gran cantidad de restos próximos entre sí que remontan, además de presentar fracturas producidas en estado seco entre otros (Lyman 1994; Villa y Mahieu 1991). Los procesos diagenéticos son producidos cuando los restos se encuentran enterrados, por un intercambio de materiales entre los huesos y el medio circundante, así como la pérdida de los componentes orgánicos de los mismos, en este caso, tal vez por fluctuaciones en la humedad del sustrato en que estaban enterrados (a esto se asocia el alto porcentaje de pátinas de óxido de manganeso). Esta pérdida de componentes orgánicos del hueso genera el debilitamiento de los restos, lo que implica una mayor fragmentación, tanto por el peso de los sedimentos, como por la recuperación del material en la excavación y la subsiguiente manipulación por el investigador (e.g. Reitz y Wing 1999; Smith et al. 2007). Por otra parte, las fracturas frescas, al no presentar atributos asociados a la fractura antrópica (e.g. hoyos de percusión, negativos de lascado), no nos permite inferir si eran o no producto de la extracción de médula.

\section{Marcas antrópicas}

Del total de los restos óseos de la muestra, el 13,51\% presenta marcas de corte $(\mathrm{N}=10)$. Siete de estas marcas son producto de actividades de descarne y se hallan presentes en tibio-tarso (Figura 3.b.1) y peroné, huesos asociados dentro de la misma región anatómica. Por otra parte, dos podrían asociarse tanto a actividades de desarticulación como de obtención de tendones, ya que se encuentran en la epífisis distal del tarso-metatarso (Figura 3.a). Finalmente una de las marcas se observan en la cara ventral de una vértebra cervical (Figura 3.c), corresponderían a actividades de descarne o cuereo (en esta región la cantidad de carne es escasa; Fernández 2010: 290 y bibliografía allí citada).
Con respecto a la confección de artefactos óseos, dos especímenes muestran evidencias de dicha práctica. Uno corresponde a un fragmento de diáfisis de tibio-tarso (Figura 4.a) con fractura postdepositacional (fractura
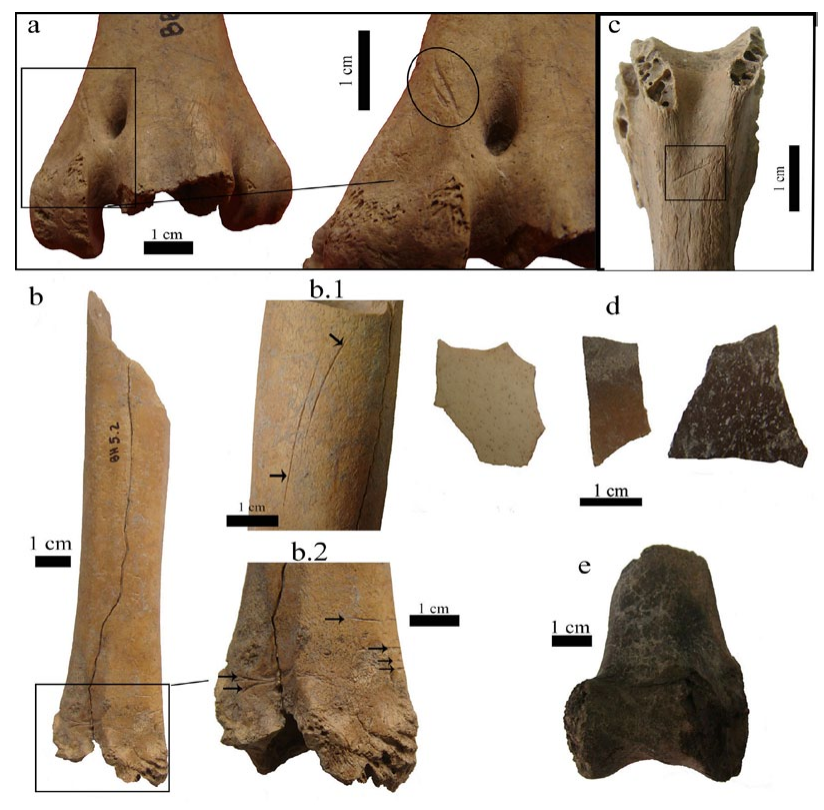

d

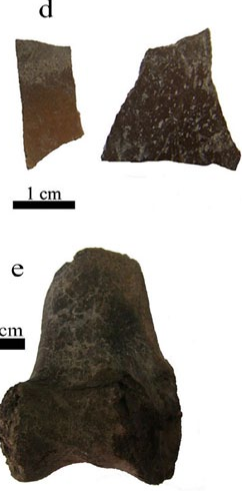

Figura 3. Marcas antrópicas en restos de $R$. americana. a: Marcas de corte en la epífisis distal de tarso-metatarso; b: Marcas de corte en diáfisis de tibio-tarso, b.1: Marcas de descarne sobre la diáfisis (cara posterior), b.2: Marcas de corte en la porción distal de la diáfisis (cara anterior); c: Marcas de corte sobre cara ventral de vértebra cervical; d: Cáscaras de huevo, imagen de la izquierda sin termoalterar, imagen del centro con bajo grado de termoalteración, imagen de la derecha con mayor grado de termoalteración; e: Porción distal de tibio-tarso carbonizada.

Figure 3. Remains of $R$. americana with anthropic marks. a: Distal epiphysis of tarso-metatarsus with cut marks; b: Diaphysis of tibiotarsus with cut marks, b. 1: fleshing marks on diaphysis (posterior side), b.2: cut marks on the distal portion of the diaphysis (anterior side); c: ventral side of cervical vertebrae with cut marks; d: eggshells, without thermal alteration (left figure), low degree of thermal alteration (central figure), high degree of thermal alteration (right figure); $e$ : distal portion of carbonized tibio-tarsus. 
longitudinal seca). Sus extremos presentan aserrado perimetral (sensu Acosta 2000), sin embargo uno presenta un mejor acabado (superficie recta regularizada por pulido Figura 4.a.1) y el otro presenta mayores irregularidades (Figura 4.a.2); a su vez, en el sector de la diáfisis cercana al extremo más irregular se observa facetado longitudinal en su cara anterior. Previamente, este artefacto fue interpretado como fragmento de tubo-ave (véase discusión de la clasificación de los tubos aves en Pérez Jimeno y del Papa 2016). El otro espécimen correspondea la mitad proximal de diáfisis de húmero, el cual presenta aserrado perimetral en su extremo proximal y fractura helicoidal-longitudinal en su otro extremo (Figura 4.b) y se considera que también pudo corresponder a un tubo-ave.

Finalmente, con respecto a la termoalteración, sólo se recuperó una epífisis distal de tibio-tarso (Figura 3.e) que se encuentra carbonizada y un bajo porcentaje de cáscaras de huevo (2,27\%, Figura 3.d) con esta característica.

\section{Discusión}

Si bien los restos de ñandú están escasamente representados en relación con otros taxones (NISP), a partir del estimador de biomasa MNI multiplicado el peso promedio del taxón en vida, estas aves quedan en el segundo lugar de importancia por su potencial aporte de nutrientes entre los recursos faunísticos. Cabe destacar, sin embargo, que existe una amplia diferencia entre el recurso de mayor importancia (los camélidos) y el segundo (reidos), con una diferencia de 57,09 puntos del

Figura 4. Artefactos óseos. 4.a: Fragmento de diáfisis de tibio-tarso con aserrado perimetral en ambos extremos, 4.a.1: Detalle de extremo con mayor regularización, 4.a.2: Detalle de extremo con menor regularización y facetado en la cara anterior de la diáfisis; 4.b: Mitad proximal de diáfisis de húmero (vista ventral), detalle de extremo proximal (vista frontal y dorsal) con aserrado perimetral.

Figure 4. Bone technology. 4.a: tibio-tarsus diaphysis fragment with edge sawing in both ends, 4.a.1: detail of the end with more regularization, 4.a.2: detail of the end with less regularization and faceted on the diaphysis front face; 4.b: proximal half of humerus mid-shaft (ventral view), detail of proximal end (frontal and dorsal view) with edge sawing.

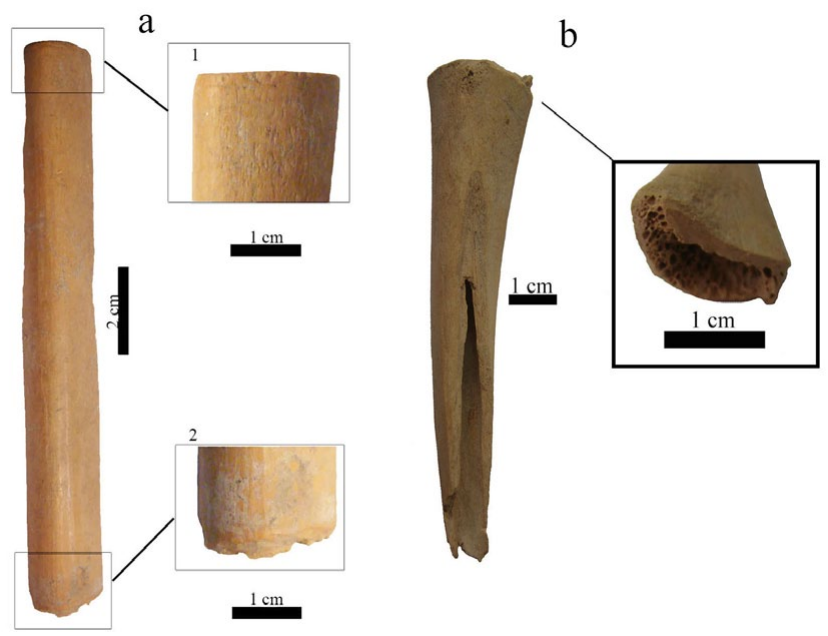

porcentaje total, lo que nos permite inferir que el ñandú fue un recurso complementario para los pobladores de BOL. La presencia de restos de cáscaras de huevo de ñandú termoalterados podría deberse a su cocción, sin embargo dicha evidencia no es conclusiva. Cabe destacar que el escaso registro de las cáscaras a través del MNI (sólo dos), hace suponer que este recurso no aportaría en demasía a la dieta. Los huevos de ñandú habrían sido recolectados principalmente entre los meses de agosto y enero, momento de la puesta de huevos de los reidos (Martella y Navarro 2006). La escasa representación de los huevos en el sitio, teniendo en cuenta que las hembras producen una media de 40 huevos por estación (datos obtenidos en condiciones de cautiverio, Martella y Navarro 2006), podría estar relacionada a diversos factores o una combinación de estos. Uno de ellos podría ser por problemas de preservación de los restos, sin embargo, por el momento no contamos con estudios actualísticos sobre el grado o intensidad en que los procesos naturales pueden afectar este tipo de material. Con respecto a la acción antrópica, varias posibilidades podrían estar en juego, una de ellas es que era un recurso obtenido de manera oportunista o con ciertas restricciones en cuanto a su utilización, o que eran consumidos en el lugar de obtención. Otra posibilidad, podría corresponder a decisiones en el manejo de la fauna silvestre con el fin de no sobreexplotarlas, ya que en silvestría sólo el 30\% de los huevos eclosiona (datos de poblaciones actuales, Martella y Navarro 2006). Si bien por el momento no tenemos evidencias de ninguna de estas posibilidades, queremos destacar que en la mayoría de los sitios de la región analizados (del Papa 2012) se observa la escasez de huevos, los cuales presentan una baja proporción de restos termoalterados, en este sentido, consideramos que esta recurrencia observada podría corresponderse con prácticas de cocción y consumo.

Con respecto a la representación diferencial de partes esqueletarias, se observa el patrón registrado para distintas regiones del país, con predominio del miembro posterior y escasez del esqueleto axial y miembro anterior (e.g. Belardi 1999; Belardi y Carballo Marina 2003; Cruz 2011; Cruz y Elkin 2003; Fernández 2010; Fernández et al. 2001; Frontini y Picasso 2010). Como contrapartida de otros casos arqueológicos (e.g. Fernández 2010), consideramos que en la muestra de estudio existe un número considerable de restos que nos permiten hacer correlaciones entre la representación anatómica y los distintos marcos de referencia analizados (véase el NISP de otros casos arqueológicos en Tabla 4). Además, los distintos pares correlacionados entre las variables nos permitieron obtener resultados significativos para algunos índices de utilidad y no significativo para la DMO. En este sentido, los resultados de las correlaciones del sitio BOL también se contraponen con lo observado por otros investigadores (Cruz y Elkin 2003; Frontini y Picasso 2010; Giardina 2010) ya que podemos inferir que la representación de partes se condice con una estrategia 
humana de tipo bulk o gourmet, donde los antiguos pobladores habrían seleccionado aquellos elementos de mayor cantidad y/o mejor calidad de nutrientes. En este sentido, los procesos tafonómicos mediados por la DMO no habrían influido de manera significativa en la conformación del perfil anatómico y por lo tanto no se observa una muestra sesgada en términos interpretativos de las Clases de Lyman (1994). Esto no quiere decir que los procesos naturales destructivos no hayan intervenido en la muestra (ver más adelante), sino que su intensidad no fue tan importante como para sesgar o dificultar la interpretación de la selección humana.

Se realizó el análisis de las modificaciones óseas con el fin de interpretar el patrón diferencial de partes esqueletarias. En este aspecto, se observa en la mayoría de los casos una nula a baja meteorización, incluso de aquellos elementos de menor DMO, lo que indicaría un enterramiento relativamente rápido de una gran proporción de los materiales. Otro aspecto importante es la ausencia de evidencias de la acción de depredadores naturales sobre los restos de ñandú. La acción de las raíces y roedores en el sitio pudieron afectar en mayor medida la distribución espacial de los restos, así como es posible que su acción pueda fragmentar algunos elementos. A su vez, tanto las raíces, los roedores y las pátinas de óxido de manganeso pudieron enmascarar marcas preexistentes. Sin embargo, consideramos que las pátinas de óxido de manganeso en forma de pequeñas manchas no influyeron en el registro de otras variables y la proporción de marcas de roedores no es considerable (cerca del 15 \%). Por último, la alta proporción de fragmentación por procesos postdepositacionales (deshidratación del hueso por diagénesis) es un aspecto a tener en cuenta y que puede afectar la proporción en que están representados los elementos, sin embargo, la gran cantidad de restos que fueron remontados mecánicamente nos permitió ser más precisos en la determinación anatómica y taxonómica de los mismos y reducir el error en la cuantificación (véase discusión en del Papa 2010, 2012).

Con respecto al procesamiento antrópico, se observaron evidencias del cuereo, descarne, desarticulación y obtención de tendones. Si bien es sugestiva la presencia de fracturas frescas y la correlación positiva y significativa entre el MAU\% y el MI, no se reconocieron atributos asociados a las fracturas que nos permitan confirmar el consumo de médula ósea. No se descarta que en este caso, la fractura postdepositacional asociada a la diagénesis de la mayor proporción de los elementos dificultara la observación de las marcas de percusión. Por otra parte, se ha reconocido la selección de elementos de ñandú con el fin de confeccionar artefactos (un tibiotarso y un húmero). Hay que tener en cuenta, que en la región de estudio se han recuperado numerosos restos de ñandú formatizados o desechos de manufactura de artefactos que se encuentran en la colección del Museo de Ciencias Antropológicas y Naturales E. y D. Wagner de
Santiago del Estero (en una visita y estudio preliminar se han reconocido principalmente tibio-tarsos, húmeros y en menor medida fémures). La selección de ciertos elementos de ñandú para confeccionar artefactos pudo haber influido en la representación de partes esqueletarias, en este caso, el único elemento del miembro anterior que se recuperó se encuentra formatizado (húmero), sin embargo, creemos que no influyó en el perfil general de partes esqueletarias, teniendo en cuenta que sólo dos especímenes presentan formatización de los 74 representados en el sitio. En las fuentes etnohistóricas es recurrente la mención del uso de las plumas de ñandú por los antiguos pobladores de la región como vestimenta (Cieza de León 1548-1551 [1877]; Fernández 1571 [1914]; Sotelo de Narváez 1583 [1885]). Si bien no se registró la presencia de plumas (principalmente por cuestiones de preservación), y son escasos los elementos del ala en el sitio, es posible que los antiguos pobladores sacaran las plumas de las alas en los lugares de obtención del recurso, transportando así, las partes de mayores nutrientes y las plumas. Por otra parte, cabe mencionar que las plumas coberteras potencialmente utilizables para la vestimenta, también se encuentran en el cuerpo, lo que podrían haber utilizado no sólo las plumas de las alas. En este sentido, consideramos que habrían utilizado al ñandú de una manera integral, aprovechando todos los productos que brinda este recurso, desde huevos, cuero, tendones, carne, grasa, médula ósea, materia prima para la confección de artefactos y posiblemente sus plumas como vestimenta.

Concluyendo, consideramos que los antiguos pobladores del sitio BOL utilizaron al ñandú como un recurso complementario de importancia (segundo puesto en relación a los otros taxones) y de una manera integral. Si bien se observa la tendencia de representación diferencial de partes esqueletarias registrado para otras regiones del país (mayor proporción de elementos del miembro posterior y escasez del esqueleto axial y miembro anterior), en este sitio se observan diferencias que nos permitieron interpretarlo como producto de un transporte selectivo y el procesamiento de los individuos. Las diferencias en los resultados aquí presentados con los obtenidos por otros investigadores, podrían estar en relación al contexto cultural del sitio de estudio. En este caso, el sitio corresponde a asentamientos de grupos sedentarios con economía mixta, tanto agrícola-ganadera (se han asignado restos a Lama cf. L. glama para sitios del mismo contexto cultural; del Papa 2012, 2015), como extractiva (cazadores, recolectores y pescadores). Además, para el momento tardío (contexto Sunchitúyoj y Averías) se infiere un aumento poblacional (Drube 2009). En este sentido, de los resultados obtenidos previamente para momentos en que se desarrolla el estilo Sunchitúyoj, se postula que ante un aumento poblacional de estos grupos sedentarios, el área alrededor del asentamiento habría sido agotada de recursos silvestres y las partidas de caza serían forzadas a recorrer distancias mayores en 
busca de recursos, por lo que los cazadores preferirían aquellas presas de mayor porte y con mayor tasa de retorno energético (véase del Papa 2012; del Papa y Togo 2015; del Papa et al. 2012), y en este caso en particular del ñandú, una selección de aquellos elementos de mayor rinde económico. Sin embargo, sería interesante poder realizar en un futuro, estudios tafonómicos actualísticos en la región de estudio con el fin de evaluar el rol de los procesos naturales y comparar con otras regiones para estimar las diferencias aquí analizadas.

La Plata, 28 de septiembre de 2016

\section{Agradecimientos}

Al profesor Juan Carlos Cejas por facilitar el trabajo de campo y su constante apoyo a los trabajos arqueológicos de la zona, a doña Elda y al Sr. Lami Hernández por permitirnos trabajar en sus propiedades y facilitar sus instalaciones, al ex intendente de Beltrán, Miguel Álvarez por el apoyo brindado. A Valeria Accinelli por la traducción del resumen y las referencias al inglés. A los evaluadores cuyos comentarios ayudaron a mejorar el manuscrito. Lo expuesto en este trabajo es responsabilidad de los autores.

Este trabajo contó con financiamiento del Proyecto del programa de Incentivos para Docentes-Investigadores, Facultad de Ciencias Naturales y Museo, UNLP, Código: N 769. Director: Dr. Luciano De Santis, y el Proyecto "Estudio antropológico integral de las poblaciones del pasado y del presente en la provincia de Santiago del Estero. Un enfoque interdisciplinario", Código 23/D190, financiado por el Consejo de Investigaciones Científicas y Tecnológicas, UNSE. Director: Dr. Hilton Daniel Drube.

\section{Bibliografía}

Acosta, A. 2000. Huellas de corte relacionadas con la manufactura de artefactos óseos en el nordeste de la provincia de Buenos Aires. Relaciones de la Sociedad Argentina de Antropología 25: 159-178.

Acosta, A. 2005. "Zooarqueología de cazadores-recolectores del extremo nororiental de la provincia de Buenos Aires (humedal del río Paraná inferior, Región Pampeana, Argentina)" . Facultad de Ciencias Naturales y Museo, UNLP, Argentina, 332 pp. Tesis Doctoral, La Plata.

Álvarez, M. C. 2015. Utilización de Rhea americana (Aves, Rheidae) en el sitio Paso Otero 4 (partido de Necochea, región pampeana). Archaeofauna 24: 53-65.

Behrensmeyer, A. K., C. T. Stayton y R. E. Chapman. 2003. Taphonomy and ecology of modern avifaunal remains from Amboseli Park, Kenya. Paleobiology 29(1): 52-70.

Belardi, J. B. 1999. Hay choiques en la terraza. Información tafonómica y primeras implicaciones arqueofaunísticas para Patagonia. Arqueología 9: 163-185.

Belardi, J. B., F. Carballo Marina. 2003. Tafonomía regional en la cuenca media del río Coyle (Santa Cruz, Patagonia Argentina).
Intersecciones en Antropología 4: 59-73.

Binford, L. R. 1981. Bones: Ancient Men and Modern Myths. Academic Press, New York.

Binford, L. R. 1984. Faunal Remains from Klasies River Mouth. Academic Press, Orlando.

Blumenschine, R. J., C. W. Marean y S. D. Capaldo. 1996. Blind test of inter-analyst correspondence and accuracy in the identification of cut marks, percussion marks, and carnivore tooth marks on bone surfaces. Journal of Archaeological Science 23: 493-507.

Bökönyi, S.1970. A new method for the determination of the number of individuals on animal bone material. American Journal of Archaeology 74: 291-292.

Bonomo, M., C. León, L. Turnes y E. Apolinaire. 2008. Nuevas Investigaciones sobre la ocupación prehispánica de la costa pampeana en el Holoceno Tardío: el sitio arqueológico Claromecó 1 (Pdo. De Tres Arroyos, Pcia. de Bs. As.). Intersecciones en Antropología 9: 25-41.

Burkart, R., N. O Bárbaro, R. O. Sánchez, y D. A. Gómez.1999. Eco-regiones de la Argentina. Administración de Parques Nacionales, Secretaría de Recursos Naturales y Desarrollo Sustentable, Presidencia de la Nación, Buenos Aires.

Carden, N., G. Martínez. 2014. Diseños fragmentados. Circulación social de imágenes sobre huevos de Rheidae en Pampa y Norpatagonia. Boletín del Museo Chileno de Arte Precolombino 19 (2): 55-75.

Cieza de León, P. Ca. 1548-1551 [1877]. Guerra civiles del Perú, Tomo II: Guerras de Chupas. Administración García Rico y Compañía, Madrid.

Clason, A. T. 1972. Some Remarks on the Use and Presentation of Archaeozoological Data. Helinium 12 (2): 139-153.

Cornaglia Fernández, J. 2013. Análisis taxonómico e inferencias paleoambientales en el sudoeste santafesino. El sitio arqueológico Laguna El Doce. Revista del Museo de La Plata, Sección Antropología 13 (87): 109-124.

Cruz, I. 2011. Tafonomía de huesos de aves. Estado de la cuestión y perspectivas desde el sur del Neotrópico. Antípoda. 13: 147-174.

Cruz, I. 2015. Las investigaciones sobre preservación de huesos de aves y mamíferos grandes en Patagonia (Argentina). Archaeofauna 24: 209-224.

Cruz, I., D. Elkin. 2003. Structural Bone Density of the Lesser Rhea (Pterocnemia pennata) (Aves: Rheidae). Taphonomic and Archaeological Implications. Journal of Archaeological Science 30: 37-44.

del Papa, L. M. 2012.“Una aproximación al estudio de los sistemas de subsistencia a través del análisis arqueofaunístico en un sector de la cuenca del Río Dulce y cercanías a la Sierra de Guasayán". Facultad de Ciencias Naturales y Museo, UNLP, Argentina, 559 pp. Tesis Doctoral, La Plata.

del Papa, L. M. 2015. First approach to study the presence 
of domesticated camelids (Lama glama) in the ChacoSantiago region, a marginal zone of the South Central Andes. International Journal of Osteoarchaeology 25: 45-60.

del Papa, L. M., L. De Santis. 2015. No se les escapó la tortuga. Uso antrópico de Chelonoidis chilensis en un sitio de la región Chaco-Santiagueña (provincia de Santiago del Estero). Arqueología 21 (1): 115-135.

del Papa, L. M., J. Togo. 2015. Estrategias de subsistencia de la etapa agroalfarera en la cuenca media del río Dulce (provincia de Santiago del Estero, Argentina). Cuadernos del Instituto Nacional de Antropología y Pensamiento Latinoamericano. Series especiales 2 (4): 106-120.

del Papa, L. M.; L. De Santis y J. Togo. 2012. Zooarqueología santiagueña. Despertando de la siesta. En A. Acosta, D. Loponte y L. Mucciolo (compiladores). Temas de Arqueología. Estudios Tafonómicos y Zooarqueológicos (II), pp. 1-24. Asociación Amigos del Instituto Nacional de Antropología, Buenos Aires.

del Papa, L. M., L. De Santis y J. Togo. 2017. Fossorial Fauna Record at BeltránOnofre Banegas-Lami Hernandez Archaeological Site (Santiago del Estero province, Argentina). A Taphonomic Approach. En M. Mondini, S. Muñoz y P. M. Fernández (eds.). Zooarchaeology in the Neotropics: Environmental diversity and human-animal interactions, pp. 137-156, Springer.

Drube, H. D. 2009. “Las poblaciones aborígenes prehispánicas de Santiago del Estero. Evaluación de sus características bioantropológicas y de sus condiciones de salud, enfermedad y nutrición."Facultad de Ciencias Naturales y Museo, UNLP, Argentina, 330 pp. Tesis Doctoral, La Plata.

Fernández, D.1571 [1914]. Primera parte de la Historia del Perú, Libro II, Colección Hispano Americana, Biblioteca Hispania, Madrid.

Fernández, P. M. 2000. Rendido a tus pies: acerca de la composición anatómica de los conjuntos arqueofaunísticos con restos de Rheiformes de Pampa y Patagonia. En Desde el país de los gigantes. Perspectivas arqueológicas en Patagonia, Tomo II, pp. 65-573-586. UNPA, Río Gallegos

Fernández, P. M. 2010. Cazadores y presas. 3.500 años de interacción entre seres humanos y animales en el noroeste de Chubut. Fundación de Historia Natural Félix de Azara, Buenos Aires.

Fernández, P. M., Cruz, I. y Elkin, D. 2001. Densidad mineral ósea de Pterocnemia pennata (Aves: Rheidae). Una herramienta para evaluar frecuencias anatómicas en sitios arqueológicos. Relaciones de la Sociedad Argentina de Antropología 26: 243-260.

Fiore, D., F. Borella. 2010. Geometrías delicadas. Diseños grabados en cáscaras de huevo de Rheidae recuperados en la costa norte del Golfo San Matías, Río Negro. Intersecciones en Antropología 11: 277-293.

Folch, A., 1992. Order Struthioniformes. En J. del Hoyo, A. Elliot y J. Sargatal (eds.). Handbook of the birds of the world, vol. 1. Ostrich to Ducks, pp. 75-110. Lynx Edit, Barcelona.

Frontini, R., M. B. Picasso. 2010. Aprovechamiento de Rhea americana en la localidad arqueológica El Guanaco. En M. Gutiérrez, M. De Nigris, P. Fernández, M. Giardina, A. Gil, A. Izeta, G. Neme y H. Yacobaccio (eds). Zooarqueología a principios del siglo XXI: Aportes Teóricos, Metodológicos y Casos de Estudio, pp: 563-574. Ediciones del Espinillo, Buenos Aires.

Giardina, M. A. 2006. Anatomía económica de Rheidae. Intersecciones en Antropología 7: 263-276.

Giardina, M. A. 2010. “El aprovechamiento de la avifauna entre las sociedades cazadoras-recolectoras del sur de Mendoza: un enfoque arqueozoológico". Facultad de Ciencias Naturales y Museo, UNLP, Argentina, 378 pp. Tesis Doctoral. La Plata.

Gómez de Silva, H., A. Oliveras de Itay R. A. Medellín Legorreta. 2005. Rhea americana. Vertebrados superiores exóticos en México: diversidad, distribución y efectos potenciales. Instituto de Ecología, Universidad Nacional Autónoma de México. Bases de datos SNIB -CONABIO. Proyecto U020. México. D.F.

González de Prado, P. 1548-1556 [1919]. Capítulos de una información de los servicios prestados por Pedro González de Prado, que entró en las provincias del Tucumán y Río de la Plata con Diego de Rojas, Felipe Gutiérrez y Nicolás de Heredia, y se señaló en la expedición de Francisco de Mendoza. Años 1548-1556. En D. R. Levillier (ed.) Gobernación del Tucumán. Probanzas de méritos y servicios de los conquistadores. Documentos del Archivo de Indias. Tomo 1, años 1548-1583, pp. 1-67. Colección de Publicaciones Históricas de la Biblioteca del Congreso Argentino, Buenos Aires.

Gramajo de Martínez Moreno, A. 1978. Evolución cultural en el territorio santiagueño a través de la arqueología. Serie Monográfica N 5. Publicación del Museo Arqueológico "Emilio Y Duncan Wagner", Santiago del Estero.

Ledesma, N. R. 1979. La verdad sobre el clima de Santiago del Estero. Cuaderno de Cultura 10 (17), Municipalidad de Santiago del Estero, Santiago del Estero.

Lizarraga, R. ca. 1604-1607 [1999]. Descripción breve del reino del Perú, río de La Plata y Chile. Union Académique Internationale, Academia Nacional de la Historia, Buenos Aires.

Lorandi, A. M. 1974. Espacio y tiempo en la prehistoria santiagueña. Relaciones de la Sociedad Argentina de Antropología 8: 199-236.

Lorandi, A. M. 1978. El Desarrollo Cultural Prehispánico en Santiago del Estero. Argentina. Journal de la Société des Américanistes 65 (1): 63-85.

Lyman, R. L. 1994. Vertebrate Taphonomy. Cambridge University Press, Cambridge.

Martella, M. B., J. L. Navarro. 2006. Proyecto Ñandú. Manejo de Rhea americana y R. pennata en la Argentina. En M. L. Bolkovic, D. Ramadori (eds.), Manejo de Fauna Silvestre en la Argentina. Programas de uso sustentable, pp: 39-50. Dirección de Fauna Silvestre, Secretaría de Ambiente y Desarrollo Sustentable, Buenos Aires.

Medina, M.; S. Pastor, E. Apolinaire y L. Turnes. 2011. Late Holocene subsistence and social integration in Sierras of Córdoba (Argentina): the South-American ostrich eggshells 
evidence. Journal of Archaeological Science 38 (9): 20712078.

Mengoni Goñalons, G. L. 1999. Cazadores de guanacos de la estepa patagónica. Sociedad Argentina Antropología, Colección tesis doctorales, Buenos Aires.

Miotti, L. 1998. Zooarqueología de la Meseta Central y Costa de Santa Cruz. Un enfoque de las estrategias adaptativas aborígenes y los paleoambientes. Revista del Museo de Historia Natural de San Rafael, 10 (1/4). Museo Municipal de Historia Natural San Rafael, Mendoza.

Miotti, L., M. Salemme. 1999. Biodiversity, taxonomic richness and specialists-generalists during Late Pleistocene/Early Holocene times in Pampa and Patagonia (Argentina, Southern South America). Quaternary International 53/54:53-68.

Muñoz, A. S., F. Savanti. 1998. Observaciones tafonómicas sobre restos avifaunísticos de la costa noreste de Tierra del Fuego. Actas y Memorias del XI Congreso Nacional de Arqueología Argentina, Revista del Museo de Historia Natural de San Rafael Tomo 20 1/2: 107-121.

Outram, A. K. 2002. Bone fracture and within-bone nutrients: an experimentally based method for investigating levels of marrow extraction. En P. Miracle, N. Milner (eds.), Consuming Passions and Patterns of Consumption, pp. 51-62. McDonald Institute for Archaeological Research, Cambridge.

Payne, S. 1975. Partial recovery and sample bias. En A.T. Clason (ed.), Archaeozoological Studies, pp. 7-17. North Holland. Amsterdam.

Pérez Jimeno, L. 2004. Análisis comparativo de dos conjuntos de artefactos óseos procedentes de la llanura aluvial del Paraná y la pampa bonaerense. En G. Martínez, M. A. Gutiérrez, R. Curtoni, M. Berón y P. Madrid (eds.), Aproximaciones Contemporáneas a la Arqueología Pampeana. Perspectivas teóricas, metodológicas, analíticas y casos de estudio, pp. 319-334. Facultad de Ciencias Sociales. UNCPBA.

Pérez Jimeno, L., L. M. del Papa. 2016. Presencia del grupo morfológico definido como Tubo-ave e hipótesis de uso. Revista de Antropología del Museo de Entre Ríos 2(1): 100-118.

Prates, L., C. Acosta Hospitaleche. 2010. Las aves de sitios arqueológicos del Holoceno tardío de Norpatagonia, Argentina. Los sitios Negro Muerto y Angostura 1 (Río Negro). Archaeofauna 19: 7-18.

Quintana, C. 2007. Cálculo del número mínimo de individuos de huevos de ñandú. Intersecciones en Antropología 9: 93-97.

Quintana, C. A., D. L. Mazzanti. 2001. Selección y Aprovechamiento de Recursos Faunísticos. En D. L. Mazzanti y C. A. Quintana (eds.), Cueva Tixi: Cazadores y Recolectores de las Sierras de Tandilla Oriental. 1 Geología, Paleontología y Zooarqueología, pp. 181-209. Laboratorio de Arqueología. Universidad Nacional de Mar del Plata. Publicación Especial 1, Mar del Plata.

Reboreda, J. C., G. Fernández. 2005. Estudios sobre la ecología del comportamiento del ñandú. Rhea americana. Publicaciones Fundación para la Conservación de las Especies y el Medio Ambiente (FUCEMA).http://www.fucema.org.ar/pdf/ fyf_comportamiento_nadu.pdf. (última consulta: 25/04/2016).

Reitz, E. J., E. S. Wing. 1999. Zooarchaeology. Cambridge University Press. Cambridge.

Ringuelet, R. A. 1961. Rasgos fundamentales de la zoogeografía de la Argentina. Physis 22: 151-170.

Rusconi, C. 1933. Instrumentos Óseos Trabajados por Indígenas Prehispánicos de Santiago del Estero. Revista de la Sociedad Amigos de la Arqueología 7: 229-251.

Salemme, M., R.Frontini, 2011. The exploitation of Rheidae in Pampa and Patagonia (Argentina) as recorded by chroniclers, naturalists and voyagers. Journal of Anthropological Archaeology 30: 473-483.

Sánchez Labrador, P. J. 1910. El Paraguay Católico, Tomo I. Imprenta De Coni Hermanos, Buenos Aires.

Santini, M.2009. "Prehistoria de la región meridional del Gran Chaco. Aportes del análisis de restos faunísticos en la reconstrucción de las estrategias adaptativas de los grupos aborígenes". Facultad de Ciencias Naturales y Museo, UNLP, Argentina, 236 pp. Tesis Doctoral, La Plata.

Scheinsohn, V. 1997. "Explotación de materias primas óseas en la Isla Grande de Tierra del Fuego", Facultad de Filosofía y Letras. UBA, Argentina. Tesis Doctoral, Buenos Aires.

Smith C. I., C. M. Nielsen-Marsh, M. M. E. Jans y M. J. Collins. 2007. Bone diagenesis in the European Holocene I: patterns and mechanisms. Journal of Archaeological Science 34: 1485-1493.

Sotelo de Narváez, P. 1583 [1885]. Relación de las provincias de Tucumán que dio Pedro Sotelo de Narváez, vecino de aquellas provincias, al muy ilustre señor Licenciado Cepeda, Presidente desta Real Audiencia de La Plata. En Relaciones Geográficas de Indias, Tomo 2. 1885, pp. 143-153. Ministerio de Fomento, Madrid.

Stiner, M. C., S. L. Kuhn, S. Weiner y O. Bar-Yosef. 1995. Differential burning, recrystalization, and fragmentation of archaeological bone. Journal of Archaeological Science 22: 223-237.

Togo, J. 2004. “Arqueología Santiagueña: Estado actual del Conocimiento y Evaluación de un Sector de la Cuenca del Río Dulce." Facultad de Ciencias Naturales y Museo, UNLP, Argentina. Tesis Doctoral, La Plata.

Togo, J. 2007. Los fechados radiocarbónicos de Santiago del Estero. Actas de resúmenes ampliados del XVI Congreso de Arqueología Argentina, Tomo 3: 227-232. San Salvador de Jujuy.

Torrella, S., J. Adámoli. 2006. Situación Ambiental de la Ecorregión del Chaco Seco. En A. Brown, U. Martinez Ortiz; M. Acerbiy J. Corcuera (eds.): La Situación Ambiental Argentina 2005, pp. 75-83. Fundación Vida Silvestre Argentina, Buenos Aires.

Villa, P., E. Mahieu. 1991. Breakage Patterns of Human Long Bones. Journal of Human Evolution 21: 27-48. 